\title{
Formation of Nanoscale Bioimprints of Muscle Cells Using UV-Cured Spin-Coated Polymers
}

\author{
Fahmi Samsuri, ${ }^{1}$ John S. Mitchell, ${ }^{2}$ Maan M. Alkaisi, ${ }^{1}$ and John J. Evans ${ }^{3}$ \\ ${ }^{1}$ Department of Electrical and Computer Engineering, University of Canterbury, Christchurch 8140, New Zealand \\ ${ }^{2}$ The New Zealand Institute for Plant \& Food Research Ltd, Hamilton 3214, New Zealand \\ ${ }^{3}$ Centre for Neuroendocrinology and Christchurch School of Medicine and Health Sciences, University of Otago, \\ Christchurch 8140, New Zealand \\ Correspondence should be addressed to Maan M. Alkaisi, maan.alkaisi@canterbury.ac.nz
}

Received 8 July 2009; Accepted 29 November 2009

Recommended by S. N. Piramanayagam

\begin{abstract}
We report a nanoscale replication method suitable for biological specimens that has potential in single cell studies and in formation of 3D biocompatible scaffolds. Earlier studies using a heat-curable polydimethylsiloxane (PDMS) or a UV-curable elastomer introduced Bioimprint replication to facilitate cell imaging. However, the replicating conditions for thermal polymerization are known to cause cell dehydration during curing. In this study, a UV-cured methacrylate copolymer was developed for use in creating replicas of living cells and was tested on rat muscle cells. Bioimprints of muscle cells were formed by spin coating under UV irradiation. The polymer replicas were then separated from the muscle cells and were analyzed under an Atomic Force Microscope (AFM), in tapping mode, because it has low tip-sample forces and thus will not destroy the fine structures of the imprint. The new polymer is biocompatible with higher replication resolution and has a faster curing process than other types of silicon-based organic polymers such as PDMS. High resolution images of the muscle cell imprints showed the micro-and nanostructures of the muscle cells, including cellular fibers and structures within the cell membranes. The AFM is able to image features at nanoscale resolution with the potential for recognizing abnormalities on cell membranes at early stages of disease progression.
\end{abstract}

Copyright () 2009 Fahmi Samsuri et al. This is an open access article distributed under the Creative Commons Attribution License, which permits unrestricted use, distribution, and reproduction in any medium, provided the original work is properly cited.

\section{Introduction}

Detection of subtle differences in cell surfaces and morphology that may be indicative of diseases and abnormalities such as cancer [1] has potential for the early diagnosis and treatment of disease and for use in biological studies. Accurate discrimination of such changes by optical microscopy remains a major challenge [2]. High-resolution microscopic techniques such as atomic force microscopy (AFM) are much better suited to such analyses as they enable threedimensional (3D) nanometer resolution. However, they have the major disadvantage of requiring high scanning tip forces that can damage the sensitive soft biological tissue. Blunter tips have been used to minimize the damage but they result in greatly diminished resolution [3]. Furthermore, scanning using a tip in an aqueous environment can cause structural movement and loss of resolution due to damping effects [4]. However, it has been predicted that AFM imaging of cells in liquid should be capable of resolutions of 50-500 nm regardless of whether the cells are living or fixed $[5,6]$. Time-consuming preparation procedures when using either air or vacuum environments associated with TEM have been a further limitation which is exacerbated by the need for dehydration and fixation, which can deform the cells and introduce artifacts in the imaging process [7]. Such nanoscale imaging tools have therefore not been widely used in life science applications. Whilst polymers have been used in the imprinting of yeasts for quartz crystal microbalance sensors $[8,9]$, they have only recently been applied to nanoscale imaging.

To overcome these imaging challenges, a polymer-based approach has been recently developed whereby the cells are coated with a monomer mixture, which is then polymerized over the cell surfaces [10-12] in a positive soft lithography technique [13]. By careful selection of the monomers it is possible to obtain an imprint of the cellular surface 
features in the polymer once the cellular material has been washed away from the replica. This imprint replica can then be imaged using high-resolution techniques such as AFM without the concerns of cellular damage. This approach has come to be known as a "Bioimprint" technique $[11,14]$. When combined with AFM imaging, continuous sampling to capture snapshots of biological events and monitoring of cell conditions can be achieved to allow studies of cellular structure and cell response to physiological and noxious stimuli. Furthermore, such techniques have potential as biomedical procedures to form 3D biocompatible and bioactive scaffolds for tissue culture.

Previous work on obtaining Bioimprints has utilized the nonbiohazardous poly(dimethylsilxoane) (PDMS) polymer composite $[10,11]$ and applied this to endometrial cancer cells [14]. This has allowed high-resolution imaging of membrane morphopological structures consistent with exocytosis. This technique utilized thermal setting of the polymer [11] and so had the disadvantage of exposing cells to very high temperatures for some minutes, followed by another curing step taking hours to complete. A UV-cured Bioimprint technique was then adopted by photocuring a siloxane copolymer to visualize pituitary cells [12]. However, there were some significant challenges to overcome with this approach, namely, distorted or permeation artifacts resulting from prolonged curing time, cell dehydration effects, an irradiation time of several minutes which had the potential to induce alterations in cell characteristics, and the large number of curing steps that were required to complete the process.

Use of methacrylic acid/ethylene glycol dimethacrylate copolymer has the advantages that the polymer will set under UV irradiation within seconds [15] if applied at appropriate volumes and polymerizes smoothly around surface features, making it potentially useful for application in Bioimprinting of nanoscale features. Furthermore, it can be spin-coated onto immobilized cells to produce polymer layers of various thicknesses.

We report the development of a new Bioimprint technique that utilizes methacrylic acid/ethylene glycol dimethacrylate copolymer to achieve rapid imprinting of nanoscale features of muscle cells. Imprints are formed through aspiration of fluid from immobilized muscle cells and application of a spin-coated polymer layer, followed by peel-off, cleaning to remove residual cellular material and imaging by AFM in tapping mode. A number of subcellular features are imprinted and the cells remain intact with a minimum of morphological changes. This imprinting process can be completed in seconds and opens the way to highly detailed AFM nanoscale imaging of a range of different cell types for the monitoring and characterization of cellular features that are associated with disease.

\section{Experimental Methods}

2.1. Cell Culture. L6 rat skeletal muscle cells at $200 \times$ $10^{3}$ cells $/ \mathrm{mL}$ or $600 \times 10^{3}$ cells $/ \mathrm{mL}$ were plated into standard 6-well culture plates each containing a glass microscope coverslips, $25 \mathrm{~mm} \times 25 \mathrm{~mm}$, previously cleaned with deionized water and $70 \%$ ethanol and grown in minimum essential medium (MEM, Gibco 12360). $500 \mathrm{~mL}$ of MEM was supplemented with L-glutamine $(5 \mathrm{~mL}, 200 \mathrm{mM}$, Gibco 25030), penicillin/streptomycin (5 mL, Gibco 15070-063), and fetal bovine serum (50 mL, Gibco 10093-144). Cultures were maintained at $37^{\circ} \mathrm{C}$, in an atmosphere containing $5 \%$ $\mathrm{CO}_{2}$. Cell growth was monitored microscopically and when cell populations on the glass slides were confluent, the slides were then ready for coating with polymer.

2.2. Polymerization onto Cells. Cell-coated coverslips were removed from medium using tweezers on the edges of the glass so that the coated cells were not removed. They were washed by immersion in $10 \mathrm{mM}$ phosphate buffered saline $\mathrm{pH}$ 7.4, followed by drying through blotting from the corners of the coverslip and then aspiration of excess fluid from the extreme edges. The coverslip was then immediately mounted into a spin-coating apparatus (Laurell Technologies Corp.) and coated with pre-prepared polymerization mixture. The polymerization mixture was composed of $1.2 \mathrm{~mL}$ of triglyme (Sigma Aldrich, St Louis, USA), $0.425 \mathrm{~mL}$ of ethylene glycol dimethacrylate, $0.0425 \mathrm{~mL}$ of methacrylic acid, and $20 \mu \mathrm{L}$ of IRGAcure 2022 (CIBA Specialty Chemicals, Basel, Switzerland) and was used immediately. Application volume was optimized at $125.5 \mu \mathrm{L}$ to give suitable coverage and thickness. The coverslip was then irradiated with an Omni Cure Series 1000 UV lamp (100 W Hg arc lamp, 27\% iris setting, $250-450 \mathrm{~nm}$ filter, EXFO Photonic Solutions Inc, Singapore) and immediately spun at $50 \mathrm{rpm}$ for $10 \mathrm{~s}$ and then continued irradiation for 15 minutes. Irradiation was conducted under an inert nitrogen atmosphere within the spin-coater to prevent reaction with oxygen.

After polymerization, the polymer easily peeled off the glass slide. Optical microscope images were collected with a CCD camera at each stage in the imprinting process and compared with images of the cells before removal from growing media. The polymer was then cleaned with deionized water followed by ultrasonication for $15-20$ s to remove cellular material from the polymer. The resulting polymer imprint could then be imaged. The soft lithography process is illustrated in Figure 1.

2.3. AFM Imaging of Polymer Imprints. A Digital Instruments (DI) 3100 Nanoscope III AFM from Veeco Instruments Inc. was used for imaging the cell samples in tapping mode. With $\mathrm{X} / \mathrm{Y}$ and Z-axial limits of $\sim 110 \mu \mathrm{m}$ and $6 \mu \mathrm{m}$, respectively, the AFM is suitable for scanning cell samples with sizes in the range of $12 \mu \mathrm{m}$ and height $<6 \mu \mathrm{m}$. All images were presented in an amber color-contrast scheme and processed using the instrument's Nanoscope III v. 5.31r1 application software.

\section{Results and Discussion}

3.1. Polymerization to Produce Imprints. To produce a polymer imprint that accurately replicates the surface features of a living cell, it is necessary to achieve polymerization rapidly 


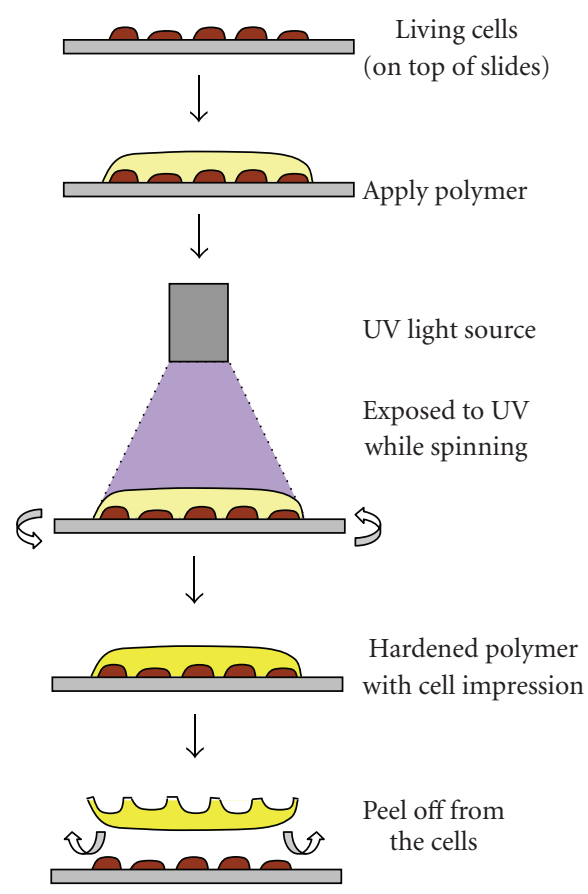

Figure 1: Schematic of the Bioimprint process showing immobilized live cells being coated with monomer mixture, polymerized under UV light, and peeled away from the glass support.

so as to minimize the time over which process-induced alterations may take place. Furthermore, the monomer solution mixture should be biologically inert so that cell response to it is minimal. These conditions must also be combined with a final polymer composition that is sufficiently flexible to mould the nanoscale features of the cell surface and not subsequently swell or shrink in such a way that these features become distorted. Our results suggest that a copolymer of MAA and EGDMA has these properties. Furthermore, as the polymer is set using rapid UV initiation polymerization, it is possible to lock in cell surface features without the need for long heat curing and the artifacts and distortions to the imprint that this brings. The UV-initiated polymerization is summarized in Figure 1.

The cell cultures were monitored to ensure the maximum number of adhered cells but at the same time not to crowd the cells to the extent that their growth and development were being hindered by other neighboring cells and to ensure minimal overlapping of the cells, which would prevent imaging of discrete cells and their surface features. It was found that once the muscle cells had been removed from media, they needed to be coated with the polymer quickly (within 5 minutes) to avoid deterioration. Prompt handling of the cells combined with rapid setting of the polymer (very thin polymer layers can set within 15-30 s; thicker layers can take 120-180 s) allowed morphology to be preserved. This could be seen in the first instance by comparing the muscle cells growing in cell culture media with cells encased in the polymer coating (Figure 2). From this, one can see that the characteristic cellular shapes have been preserved in the polymer. Circular features in the growth medium and

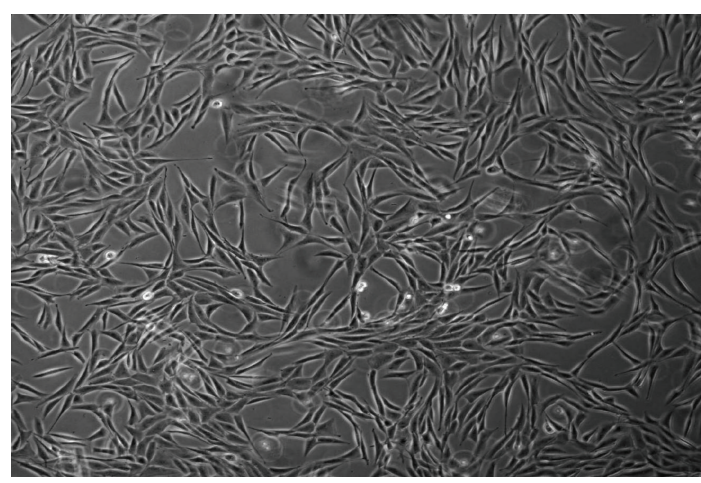

(a)

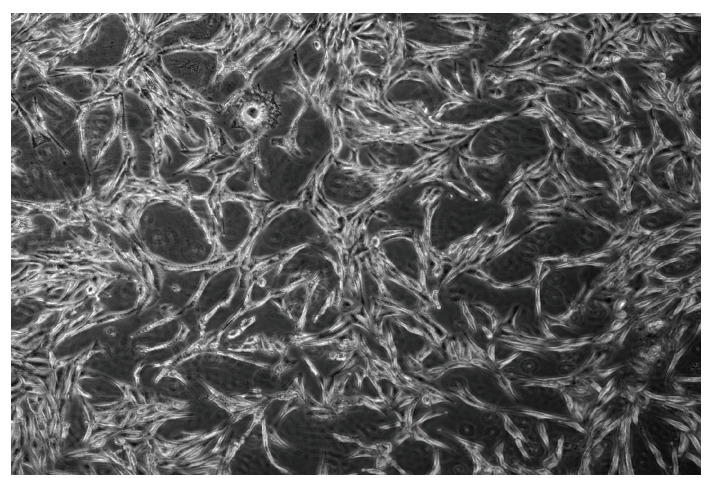

(b)

Figure 2: Light microscope image at $20 \times$ magnification showing L6 rat muscle cells (a) in culture medium before imprinting and (b) after imprinting with methacrylic acid/ethylene glycol copolymer coating (which is transparent) in place.

polymer matrix are small accretions of salt or dust and can be removed in the washing process.

The polymer matrix is largely composed of ethylene glycol units, and indeed polyethylene glycol polymers are well known for being nontoxic and nonimmunogenic [16] and are used in a number of clinical products, including skin cream. This is likely to confer low toxicity on the polymer as a whole and further aid the preservation of cellular features. Low spin speeds were applied to ensure an even coating across the surfaces of the cells but not high enough to cause significant spin-off of the polymerizing matrix. The triglyme was added to boost the viscosity of the polymer and to act as a porogen. Thick coatings of this polymer are inflexible and quite firm, which makes it easy to break if not handled carefully and it is not as malleable as PDMS. The amount of fluid in the layer between the cell and the polymer after aspiration affects the quality of the imprint; if it is too high it will cause degradation in resolution transfer of cell topography by diluting the monomer mixture and interfering with the intimate contact of the polymer solution with the cell surface.

High UV irradiation strength is required to ensure rapid setting of the polymer. Polymer curing times depend upon the volumes of polymerization mixture applied, with the $125.5 \mu \mathrm{L}$ application volume taking $120-180 \mathrm{~s}$. Irradiation 


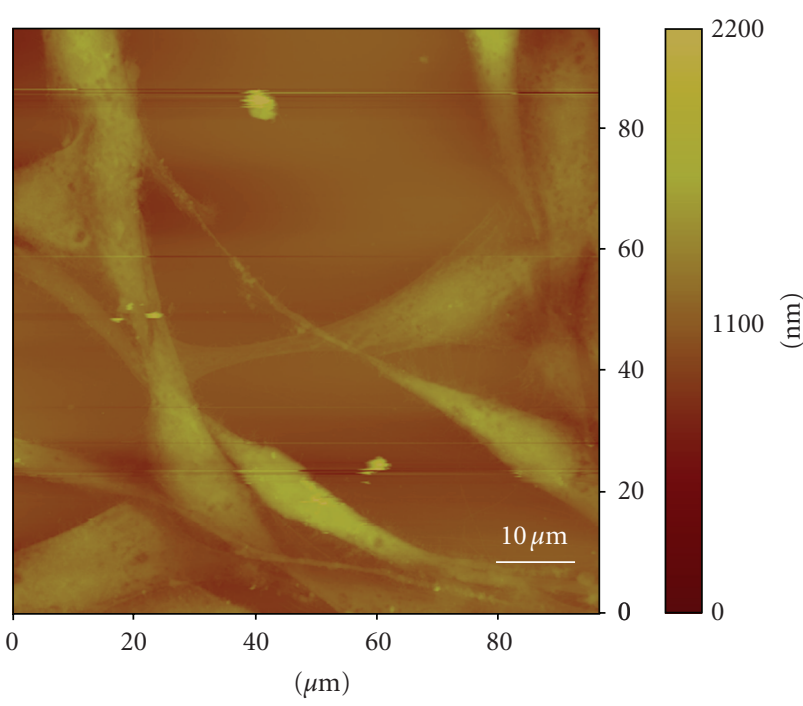

Imprint showing cultured whole cells

(a)

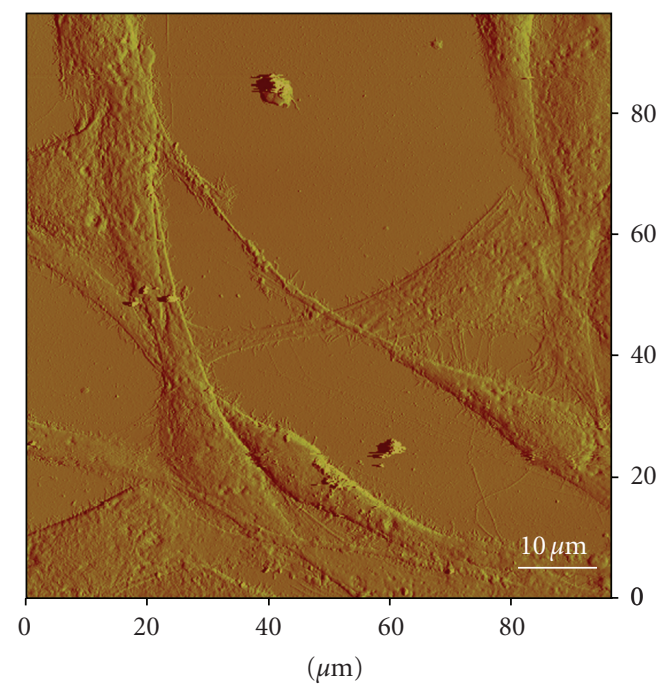

Image of cells obtained in amplitude

(b)

FIgURE 3: Atomic force microscope (AFM) images of the MAA/ EGDMA polymer imprints of rat muscle cells.

was continued for a total of 15 minutes to ensure maximum polymer hardness so that the polymer was suitable for use with an AFM tip and to help eliminate any remaining polymer free radicals. A slow, long time curing process would probably affect the impression when rapid cellular processes are occurring and so it is possible that fast UV curing may have a better chance of realizing snapshots of these processes as they occur. Muscle cells were chosen as a model in this case as they have excellent adherence to solid surfaces, which is especially important for coating on glass, which has been found to be a difficult matrix for immobilization of cells. Furthermore, the L6 cell line is commonly used in the study of tissue development and the study of the effects of nutrition and exercise on muscular development.

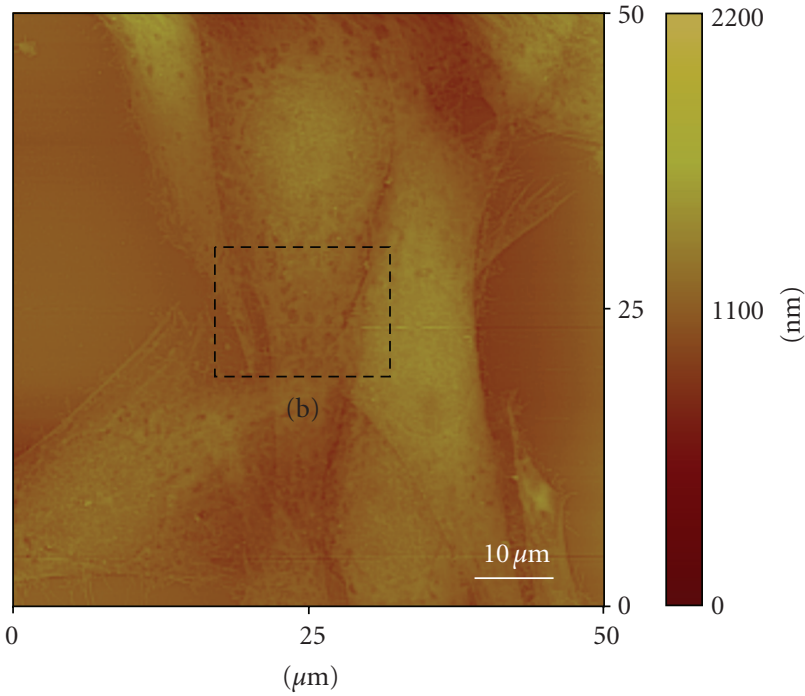

Several cultured cells with abutting plasma membranes

(a)

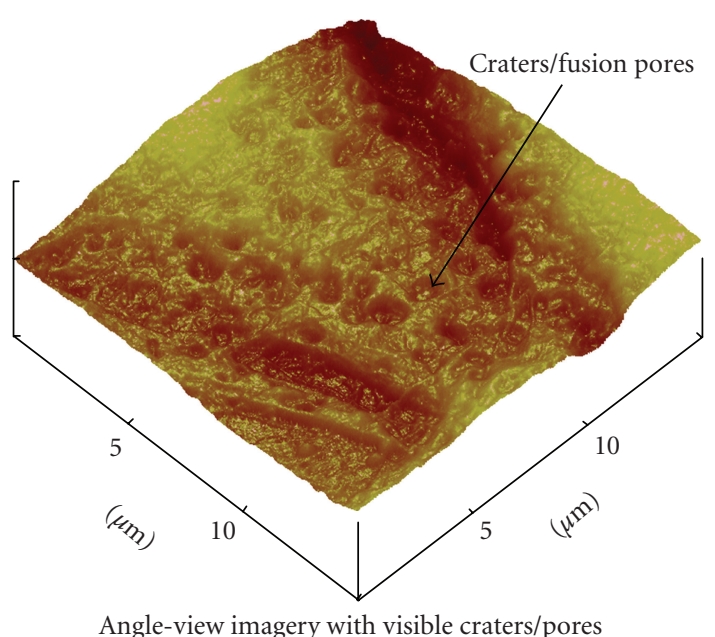

(b)

FIgURE 4: Atomic force microscope (AFM) images showing the topography and morphology of the polymer imprints of L6 rat muscle cells.

3.2. AFM Imaging of Cellular Features. Figures 3, 4, and 5 show the top view and angle view images of the muscle cells captured using the AFM. Figure 3(a) clearly shows the topography and the surface structure of the cells, while Figure 3(b) shows that the polymer replica has captured at the nanometer scale detailed features consistent with a functioning cell. At higher magnifications the range of membrane topography is observable. Structures that resemble fusion pores are shown in Figure 4. In Figure 4(a), the replica of several cultured cells with abutting plasma membranes are highly visible. The angle-view imagery in Figure 4(b) highlighted craters which are thought to be a result of fusion pores forming on the membrane by underlying granules. Our hypothesis is that these craters 


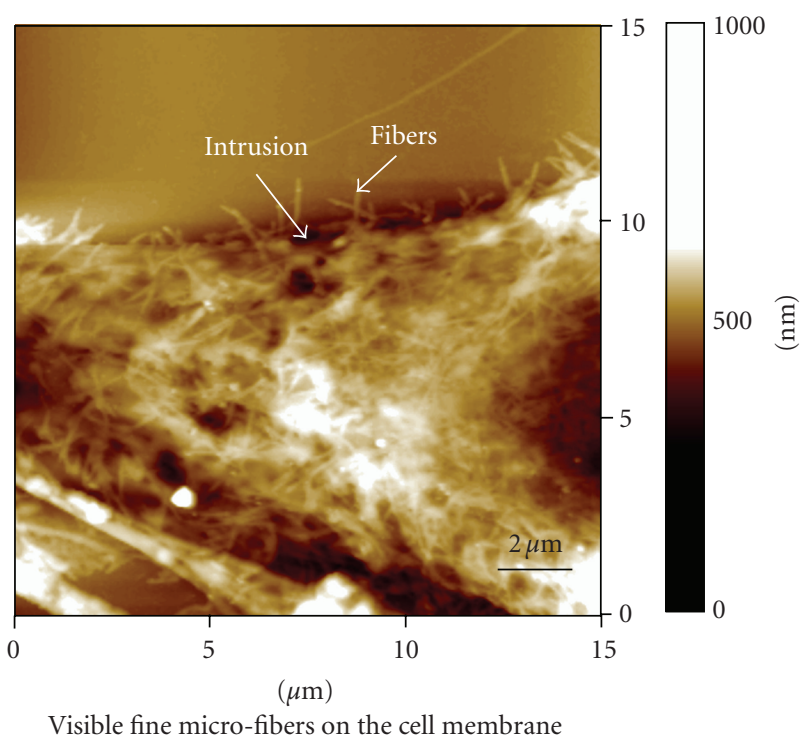

(a)

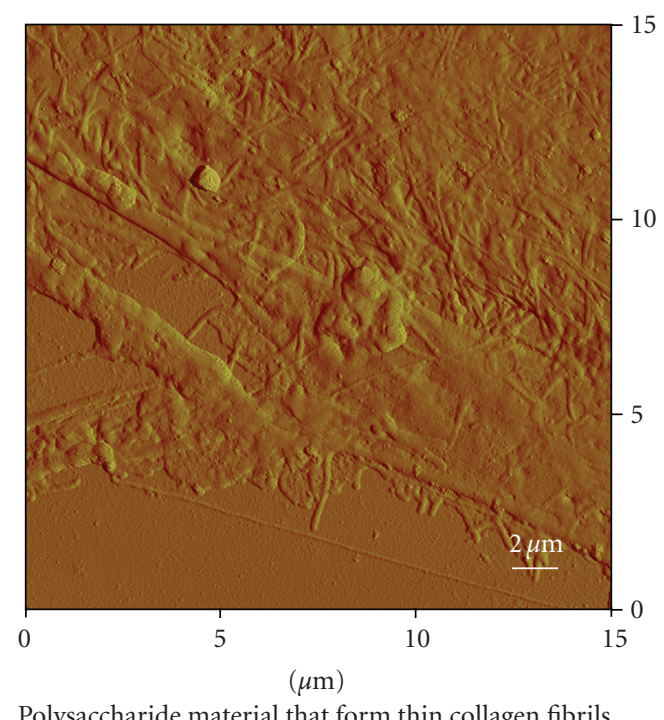

(b)

Figure 5: Images of cell intrusions and fibers illustrating the presence of ribosome on the membrane in muscle cell imprints.

are steps on a transitional series of sequential structures that represent either an endocytotic or exocytotic process. On the other hand, the possibility that these structures have other functions or they are cell permeation artifacts resulting from the polymerization process cannot be ruled out.

In addition, the polymer has also succeeded in replicating the cell submembraneous structures. Figure 5 depicts elements on the sarcolemma of the cell, which is the cell membrane of a muscle cell.

The Bioimprint resolves numerous thin collagen fibrils in the form of fine fibers that extend from the outer coat of the membrane, which is made of a thin layer of polysaccharide material, as shown in Figure 5(a). At each end of the muscle fiber, this surface layer of the sarcolemma fuses with a

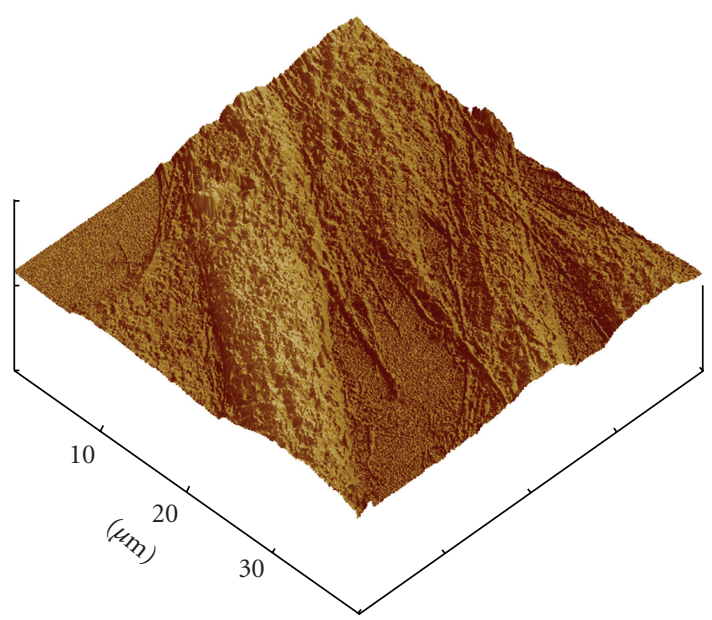

FIgURE 6: Atomic force microscope (AFM) contour image of L6 rat muscle cell imprint showing microfibrils at the ends of the muscle cells.

tendon fiber, and the tendon fibers in turn collect into bundles to form the muscle tendons that then insert into bones. The membrane is designed to receive and conduct stimuli. Both Figures 5(a) and 5(b) also display the presence of a structured cytoskeleton consisting of the intermediate cytoplasm filament proteins along with actin filaments. Actin filaments attach to the sarcolemma by focal adhesion in a spiral corkscrew fashion, and contractile proteins can organize into zones of actin and myosin along the axis of the cell.

Figure 6 shows a 3D AFM image of the muscle cell, illustrating the preserved morphology of the cell after the imprinting process. There was no evidence of any damage to the imprint from the tip of the AFM probe such as tears or scratches in the imprint. This indicates that the polymer is firm enough to resist abrasion during the microscopic imaging process and thus is suitable for use in imaging cells on the nanoscale.

Fast UV irradiation hardens the polymer sufficiently so that the replica is ready for AFM imaging of the cellular structure within 15-30 seconds. However, whether it can capture cellular processes such as steps in exocytotic fusion which may last only a few milliseconds, remains unknown as yet. Therefore, more testing is required to ascertain the limitations of the impression in accurately representing the dynamic structures of a living cell.

\section{Conclusion}

The use of a simple UV-initiated coating of a methacrylic acid/ethylene glycol dimethacrylate copolymer allows the formation of an accurate imprint of muscle cells with features resolved to the nanoscale by use of AFM imaging without damage to the imprint. This technique that has been developed has the further benefits of fast curing, thus minimizing any morphological changes to the cells, ease of use, and sufficiently low cytotoxicity. Nanoscopic 
imaging suggests that cellular structures have apparently been retained; however, at present total aspiration of liquid from the immobilized cells is required for imprinting which might cause some stress to the cells.

The imprints thus formed allow visualization of nanoscale features, such as various sizes of intrusions and pores on the surface of the cells. Minuscule delicate extrusions of molecular fibers can also be seen branching from the cell membrane. These results would suggest that the polymer has succeeded in replicating the intricate and nanoscale cellular structures of the muscle cells and provides a viable alternative to harsher chemical fixative techniques. Further work will focus on the imaging of dynamic cellular processes such as exocytotic fusion and on applying the polymer to different types of cells for applications including early cancer diagnosis.

\section{Acknowledgments}

The authors thank Robyn Wells and Peter Sykes for assistance with cell culture and Dr. Roger Hurst for input on cellular biology. This work was supported by the MacDiarmid Institute for Advanced Materials and Nanotechnology, University of Canterbury, Christchurch and by The New Zealand Institute for Plant \& Food Research Ltd.

\section{References}

[1] G. N. Papanicolaou and H. F. Traut, "The diagnostic value of vaginal smears in carcinoma of the uterus," American Journal of Obstetrics \& Gynecology, vol. 42, no. 2, pp. 193-206, 1941.

[2] L. Hamby, "Gene expression patterns and breast cancer," Cancer Genetics News, vol. 4, p. 1, 2002.

[3] H. X. You and L. Yu, "Atomic force microscopy imaging of living cells: progress, problems and prospects," Methods in Cell Science, vol. 21, no. 1, pp. 1-17, 1999.

[4] W. Häberle, J. K. H. Hörber, and G. Binnig, "Force microscopy on living cells," The Journal of Vacuum Science \& Technology B, vol. 9, no. 2, pp. 1210-1213, 1990.

[5] M. Fritz, M. Radmacher, and H. E. Gaub, "Granula motion and membrane spreading during activation of human platelets imaged by atomic force microscopy," Biophysical Journal, vol. 66, no. 5, pp. 1328-1334, 1994.

[6] M. Radmacher, "Measuring the elastic properties of biological samples with the AFM," IEEE Engineering in Medicine and Biology Magazine, vol. 16, no. 2, pp. 47-57, 1997.

[7] A. Garg and E. Kokkoli, "Characterizing particulate drugdelivery carriers with atomic force microscopy," IEEE Engineering in Medicine and Biology Magazine, vol. 24, no. 1, pp. 87-95, 2005.

[8] F. L. Dickert and O. Hayden, "Bioimprinting of polymers and sol-gel phases. Selective detection of yeasts with imprinted polymers," Analytical Chemistry, vol. 74, no. 6, pp. 1302-1306, 2002.

[9] K. Seidler, P. A. Lieberzeit, and F. L. Dickert, "Application of yeast imprinting in biotechnology and process control," Analyst, vol. 134, no. 2, pp. 361-366, 2009.

[10] M. M. Alkaisi, J. J. Muys, and J. J. Evans, "Bioimprint replication of single cells on a biochip," in BioMEMS and Nanotechnology III, vol. 6799 of Proceedings of SPIE, Canberra, Australia, December 2007.
[11] J. J. Muys, M. M. Alkaisi, and J. J. Evans, "Nanoscale analysis by replication of cellular topography using soft lithography," Journal of Biomedical Nanotechnology, vol. 2, no. 1, pp. 11-15, 2006.

[12] J. J. Muys, M. M. Alkaisi, and J. J. Evans, "Cellular replication and atomic force microscope imaging using a UV-bioimprint technique," Nanomedicine, vol. 2, no. 3, pp. 169-176, 2006.

[13] G. M. Whitesides, E. Ostuni, S. Takayama, X. Jiang, and D. E. Ingber, "Soft lithography in biology and biochemistry," Annual Review of Biomedical Engineering, vol. 3, pp. 335-373, 2001.

[14] J. J. Muys, M. M. Alkaisi, D. O. Melville, et al., "Cellular transfer and AFM imaging of cancer cells using Bioimprint," Journal of Nanobiotechnology, vol. 4, article 1, 2006.

[15] R. H. Schmidt, K. Mosbach, and K. Haupt, "A simple method for spin-coating molecularly imprinted polymer films of controlled thickness and porosity," Advanced Materials, vol. 16, no. 8, pp. 719-722, 2004.

[16] F. M. Veronese and A. Mero, "The impact of PEGylation on biological therapies," BioDrugs, vol. 22, no. 5, pp. 315-329, 2008. 

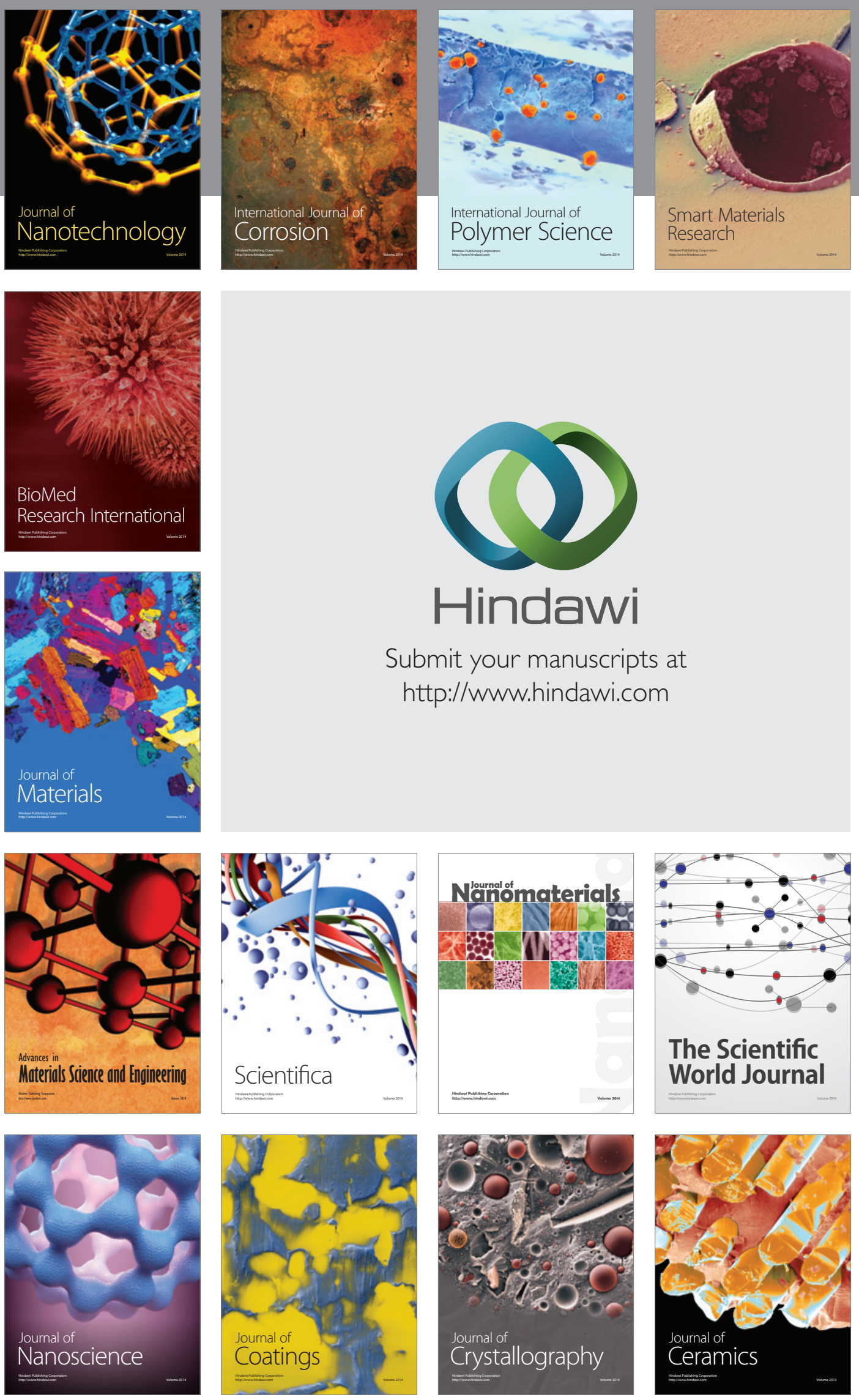

The Scientific World Journal

Submit your manuscripts at

http://www.hindawi.com

\section{World Journal}

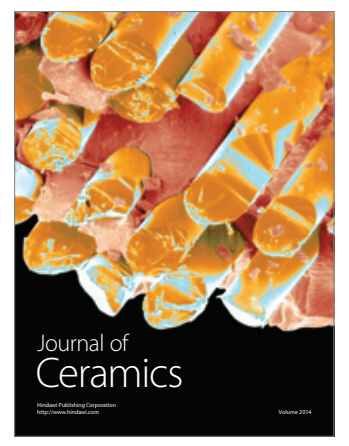

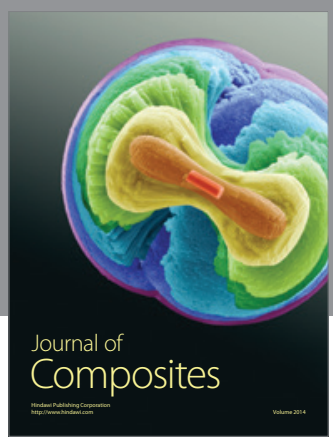
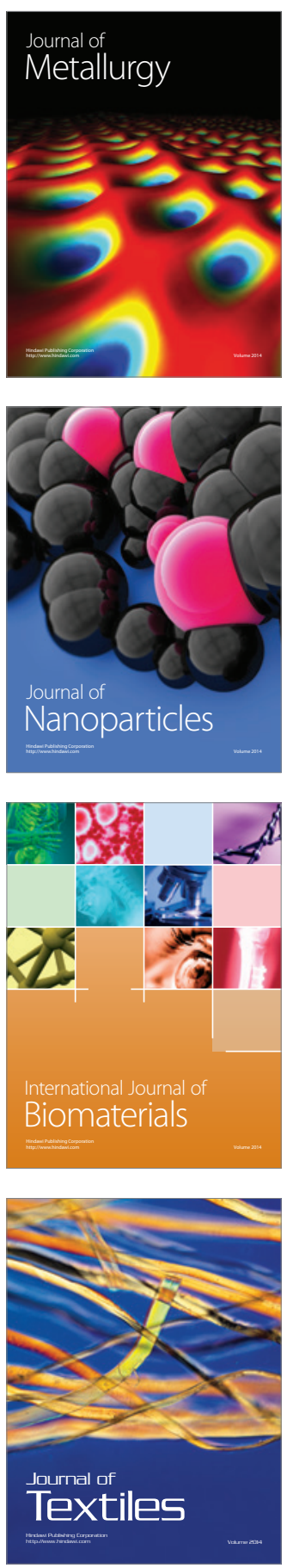